\title{
Erratum: "Electric Control of Magnetic Moment in a Ferromagnet/Semiconductor Hybrid System" [JETP Lett. 78 (9), 564 (2003)]
}

\section{L. Korenev}

PACS numbers: 85.35.Be; 85.75.-d; 99.10.Cd

In Eq. (2), $J p$ should be replaced by $J g$.

P. 565, right column, 6th line from bottom should read "coupling parameter $K \equiv J^{2} g / 4 \beta<1$."

P. 567, left column, 15th line from bottom should read " $5 \times 10^{8} \mathrm{~Hz}$." 